\title{
BMJ Open Efficacy of hand-arm bimanual intensive therapy including lower extremities (HABIT-ILE) in young children with bilateral cerebral palsy (GMFCS III-IV) in a low and middle-income country: protocol of a randomised controlled trial
}

Emmanuel Segnon Sogbossi (D , , ${ }^{1,2}$ Solange Sotindjo Adon, ${ }^{3}$ Leontine Adjagodo, ${ }^{4}$ Solange Dossou, ${ }^{4}$ Hyppolite Dakè, ${ }^{5}$ Daniela Ebner-Karestinos, ${ }^{1}$ Rodrigo Araneda (D) , ', Geoffroy Saussez, ${ }^{6}$ Julie Paradis, ${ }^{7}$ Toussaint G Kpadonou, ${ }^{2,5}$ Yannick Bleyenheuft ${ }^{1}$

To cite: Sogbossi ES, Sotindjo Adon S, Adjagodo L, et al. Efficacy of hand-arm bimanual intensive therapy including lower extremities (HABIT-ILE) in young children with bilateral cerebral palsy (GMFCS III-IV) in a low and middle-income country: protocol of a randomised controlled trial. BMJ Open 2021;11:e050958. doi:10.1136/ bmjopen-2021-050958

- Prepublication history for this paper is available online. To view these files, please visit the journal online (http://dx.doi org/10.1136/bmjopen-2021 050958).

Received 06 March 2021 Accepted 17 September 2021

D) Check for updates

(C) Author(s) (or their employer(s)) 2021. Re-use permitted under CC BY-NC. No commercial re-use. See rights and permissions. Published by BMJ.

For numbered affiliations see end of article.

\section{Correspondence to} Dr Yannick Bleyenheuft; Yannick.bleyenheuft@uclouvain. be

\section{ABSTRACT}

Introduction Cerebral palsy (CP) is highly prevalent in sub-Saharan Africa, where clinically-based studies have shown a considerable over-representation of the severe bilateral subtype. However, children's access to rehabilitation care is limited by many local factors, notably the lacking of rehabilitation services, insufficient knowledge of caregivers and financial constraints. In such a context there is an urgent need for studies of the evidence-based rehabilitation approach. Here, we describe the protocol of a randomised controlled study to investigate the efficacy of Hand-Arm Bimanual Intensive Therapy Including the Lower Extremities (HABIT-ILE) in young children with bilateral CP in Benin Republic, a representative low and middle-income country of western Africa.

Methods and analysis Forty children with bilateral CP aged between 24 and 59 months and with level III-IV in the gross motor function classification will be randomised to either a high intensity conventional therapy or HABITILE therapy. Both therapies will be delivered as a daycamp model over 2 weeks to a total of 50 hours ( 5 hours per day). The assessor-blinded primary outcomes will include the gross motor function measure and both hands assessment. Secondary outcomes will be the adapted version of the ACTIVLIM-CP questionnaire, the Canadian Occupational Performance Measure, and a perception of $\mathrm{CP}$ interview form. Children will be assessed at baseline, after intervention and at 6-week follow-up. A 2 (group) $\times 3$ (test sessions) repeated analysis of variance will evaluate changes after the interventions.

Ethics and dissemination This study has been approved by the ethics committee of the rehabilitation department of the National Teaching Hospital Hubert Koutoukou Maga of Cotonou, Benin (approval decision: N01-2019/MS/CNHUHKM/CEI/CUMPR). All participants' parents/caregivers will provide their written informed consent. Data will be managed with confidentiality.

Trial registration number PACTR201911894444879.
Strengths and limitations of this study

This is a randomised controlled trial conducted in a developing country to test the efficacy in improving motor and daily life outcomes of an intensive rehabilitation approach, the Hand-Arm Bimanual Intensive Therapy Including the Lower Extremities (HABIT-ILE) in young children with moderate and severe bilateral cerebral palsy.

- The HABIT-ILE approach will be compared with conventional therapy at the same high intensity of 50 hours (5 hours a day for 10 consecutive workdays).

- Primary outcomes (gross motor and bimanual functions) will be video-recorded and scored offline by certified examiners blinded to treatment group.

\section{BACKGROUND}

Cerebral palsy (CP) is a group of motor development disorders resulting from a permanent injury of the immature brain. Often accompanied by impairments in sensory, communication, cognitive and musculoskeletal functions, CP brings serious limitations to the autonomy of affected children in their daily life activities. ${ }^{12}$ According to the topographical distribution of the motor impairments, $\mathrm{CP}$ can be broadly classified into two groups: unilateral CP affecting mainly one side of the body, and bilateral CP affecting both sides. ${ }^{3}$ The severity of the motor impairments is commonly assessed with the gross motor function classification system (GMFCS), ranging from level I (independent ambulation) to level $\mathrm{V}$ (transported in a manual wheelchair in all settings). ${ }^{13}$ The prevalence of CP in sub-Saharan Africa is estimated to be in the 
range of 2.4 to 3.6 per 1000 children. ${ }^{4}$ Moreover, up to $70 \%$ of children with CP admitted in clinical settings in sub-Saharan Africa present the bilateral type of CP, and are classified as having moderate-to-severe impairment, that is, levels III to V of the GMFCS. ${ }^{5-7}$

Progress in the paediatrics rehabilitation field during the last decades, has led to investigations mainly in highincome countries of intensive therapies based on motor skill learning principles, which have shown promising efficacy to improve the motor function in children with $\mathrm{CP}^{8}{ }^{8}$ The relevant motor skill learning principles call for high repetition of child-initiated active movements, a progressive increase of task difficulty or complexity (shaping), a motivating environment entailing tasks of a child-friendly nature and positive reinforcements from therapists. ${ }^{8-10}$ Moreover, these therapies are directed towards attaining functional goals defined in consultation with children and parents. Following a programme embodying these principles demonstrably elicits neuroplastic changes in the brain, which underlie the motor/functional outcomes. ${ }^{1112}$ The intensive form of motor-skill learning based therapies is generally delivered at a high dosage totalling 60-90 hours in 2-3 weeks. ${ }^{13}$ The Hand-Arm Bimanual Intensive Therapy Including the Lower Extremities (HABIT-ILE) is a motor skill learning-based therapy that targets the upper and lower extremities as well as postural control. ${ }^{9}$ It has demonstrated a capacity to improve upper and lower extremities outcomes in children with either unilateral or bilateral CP, when implemented in high-income countries. ${ }^{14-17}$ Currently, studies with large sample sizes are investigating the effect of HABIT-ILE in children with bilateral CP in Australia and Europe. ${ }^{18-20}$

However, the rehabilitation context in sub-Saharan Africa is distinct from the case of high-income countries, largely due to limitations in the access to rehabilitation care of children with CP. Studies focusing on this issue have reported on the detrimental effects of socioeconomic status, lack of rehabilitation services and inadequate knowledge of caregivers, to name but a few relevant factors. ${ }^{21-23}$ Those specific issues are intertwined with the general burden of raising a child with $\mathrm{CP}$ in sub-Saharan Africa. Indeed, parents with limited incomes have difficulties in providing rehabilitation care for their child in institutional services. Furthermore, these services are often located far from home, which limits accessibility for children with CP in measure of their severity level. Consequently, parents may end up abandoning the few existing rehabilitation services. ${ }^{21} 2324$ These difficulties in raising a child with CP in sub-Saharan Africa likely explain the high rate of early mortality mostly in children with severe CP (GMFCS levels IV and V). ${ }^{45}$ In addition, two complementary reviews on CP in Africa have documented the general lack of studies on rehabilitation approaches. ${ }^{26}{ }^{27}$ Such studies as are available mainly focus on the aspects of epidemiology, socio-cultural and quality of life aspects; there is a conspicuous lack of information about evidence-based rehabilitation approaches for early intervention in the context of sub-Saharan Africa.
Therefore, the aim of this randomised controlled trial is to investigate the efficacy of HABIT-ILE in improving the motor function and daily life activities in young children with moderate-to-severe bilateral CP (GMFCS levels III and IV), as compared with the conventional therapy usually delivered to children with CP at the same high dosage in Benin, a representative low and middle-income Country in Western sub-Saharan Africa. We predicted that a 2-week goal-oriented HABIT-ILE intervention would be better tolerated by children and parents and show greater efficacy.

\section{Aims and hypotheses}

\section{Primary aim}

This randomised controlled trial will test the hypothesis that the intensive HABIT-ILE training is more efficacious than the similarly intensive programme of conventional therapy in improving gross motor function (assessed with the gross motor function measure, GMFM-66) and upper extremity function (assessed with the both hands assessment, BoHA) in children with bilateral CP aged from 24 to 59 months, with benefits evident at the conclusion of the intervention and persisting to 6 weeks follow-up.

\section{Primary hypothesis}

HABIT-ILE and conventional therapies delivered at the same high dosage will similarly improve gross motor function (GMFM-66) and bimanual manipulation (BoHA) of children with bilateral CP, but with greater improvement in the HABIT-ILE group.

\section{Secondary aims and hypotheses}

Aim: to investigate the effects of the two interventions on daily life activities, as assessed with the Canadian Occupational Performance Measure (COPM) and an adapted version of ACTIVLIM-CP questionnaire.

Hypothesis: HABIT-ILE therapy will improve daily life activities to a greater extent than conventional therapy.

Aim: to investigate the effect of both interventions on caregivers' perceptions of CP through a semi-structured questionnaire-like interview about their knowledge of CP (causes, treatment approaches and developmental outcomes) and the impact of caregiving on social and family life.

Hypothesis: parents of children in the HABIT-ILE group will have a more accurate perception of $\mathrm{CP}$ after the intervention compared with those in the conventional therapy group.

\section{METHODS}

\section{Study design}

This study is a randomised controlled trial with blindedexaminers on main outcomes to be conducted in the Republic of Benin, a low and middle-income country in Western sub-Saharan Africa, to compare the efficacies of high dosage interventions with HABIT-ILE versus conventional therapy in young children with bilateral 


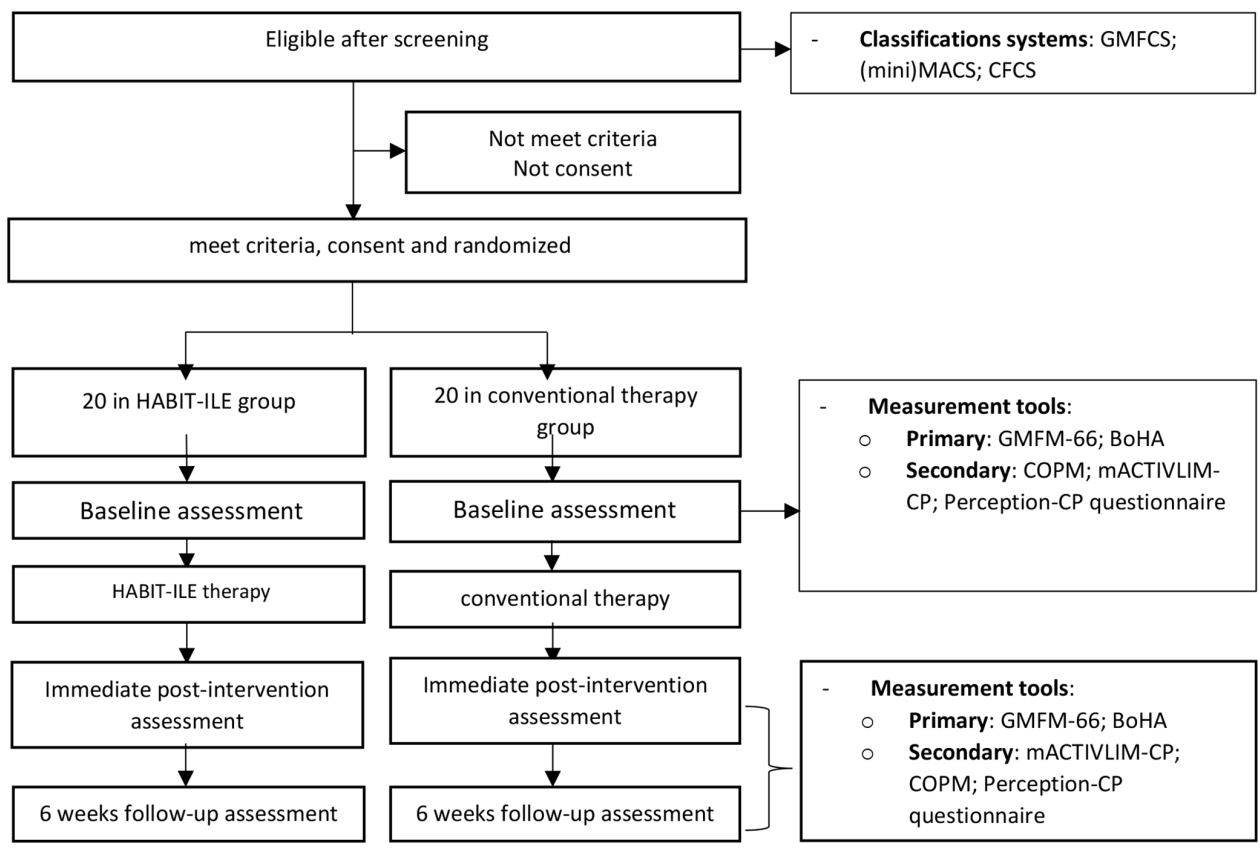

Figure 1 Consolidated Standards of Reporting Trials diagram. BoHA, both hands assessment; CP, cerebral palsy; CFCS, Communication Function Classification System; COPM, Canadian Occupational Performance Measure; GMFCS, Gross Motor Function Classification System; GMFM-66, Gross Motor Function Measure 66 items; HABIT-ILE, Hand-Arm Bimanual Intensive Therapy Including Lower Extremities; MACS, Manual Ability Classification System; mACTIVLIM-CP: modified version of ACTIVLIM-CP questionnaire.

CP. Children will be assessed at baseline, immediately after conclusion of the programme, and 6 weeks postintervention. The study design is presented in figure 1 .

\section{Recruitment}

Forty children with a diagnosis of bilateral $\mathrm{CP}$ and aged from 24 to 59 months will be included. Children will be recruited from community-based rehabilitation centres and rehabilitation departments of hospitals in Cotonou (a city of some 680000 in the South of Benin). A comprehensive consent document will be given to parents to sign if they agree to their child's participation. Details of participants' recruitment are presented in figure 1.

Inclusion criteria: To be included in the study, children must be: (1) diagnosed with bilateral CP (diplegia, triplegia or quadriplegia), (2) aged 24-59 months, (3) classified as level III or IV in the GMFCS, expanded and revised, (4) classified as level I to III on the (mini) Manual Ability Classification System (MACS) and (5) able to follow instructions and complete the assessments.

Exclusion criteria include: (1) uncontrolled seizures, (2) severe vision impairments that could interfere with the assessment and treatment and (3) any treatment over the last 6 months that might interfere with the study.

\section{Sample size}

In a recent study, Bleyenheuft et al, ${ }^{14}$ showed an improvement in gross motor function (GMFM-66) in Belgian and American children with bilateral CP immediately after HABIT-ILE therapy. The mean change (SD) in GMFM-66 was 7 (6.4) \% of logits. Assuming alpha $=0.05$ and a statistical power of $80 \%$, the sample size required was 13 children per group. Considering potential dropouts, we will include 20 children per group.

\section{Randomisation process}

Children will be paired according to their GMFCS (III/ IV), MACS (I-II/III) and age (2/3/4 years) at inclusion. Children will be randomly allocated to either the HABIT-ILE or conventional therapy group using the web site www.randomizer.org. The randomisation will be performed by the first author (ESS).

\section{Blinding procedure}

The primary outcomes measures, GMFM-66 and BoHA, will be administered by trained/certified assessors and video-recorded. The videos will be coded and scored offline by certified examiners who are blinded to treatment group. Data will be anonymised and stored by the first author (ESS) until the end of the study.

\section{Study interventions}

HABIT-ILE

HABIT-ILE is a motor learning-based approach that targets concomitantly bimanual coordination and the lower extremities, as well as postural trunk control. ${ }^{9}$ It is performed with structured activities/games adjusted to the child's motor abilities, and entails a large number of active movements repetitions with a progressive increase in difficulty. Activities are child-friendly, delivered in a motivating environment along with constant positive reinforcement from interventionists, and an amusing and engaging nature of the proposed activities/games. In addition, functional goals are defined in consultation with 
parents of the young children before the intervention, thus identifying daily activities that the children desire to perform, but have difficulty in doing so. These functional goals, demonstrated through videotape recordings by the parents, are analysed by therapy supervisors experienced with HABIT-ILE, who then tailor the therapy for each child according to his/her present motor abilities and aspirations (where to start and how to progress?). The therapy is 'hands-off', thus encouraging the child to make active movements to establish new skills. Whole-task and partial-task practices are also used. The activities/games are discussed with interventionists and supervisors on a day-to-day basis to (re)adjust and calibrate the therapy for each child.

As described by Bleyenheuft and Gordon, ${ }^{9}$ activities can be grouped in:

1. Upper extremities bimanual task activities are selected to elicit bimanual use (eg, building a pot with clay), and which include reaching, stabilising, holding, grasping and manipulation subtasks. Progression of task difficulty can start with the more affected upper extremity serving mainly as a stabiliser, while the less affected upper extremity is used for manipulating (eg, holding the paper during painting), proceeding towards a more active assisting role (eg, holding the pestle with both hands to crush material in a mortar) or toward a manipulator role requiring gross dexterity (eg, transferring building blocks to the less affected hand in building activities) or fine dexterity (eg, crafting a necklace with small beads). The activities/games are mainly performed on a table with the child sitting on a bench to place a lesser demand on trunk/postural control. However, to increase demands on postural/ trunk control, children can be initially sitting on a deflated ball during less demanding bimanual activities, with progressive inflation of the ball to increase the demand for postural control.

2. Lower extremities and trunk control activities include mainly gross motor activities/games engaging the upper/lower extremities and the trunk such as rising from the ground with/without support from furniture, throwing a toy car from the table to a race track on the floor, mobility activities such as crawling, walking with an assisting device and transfer activities (proceeding from sitting to standing). Progression in task difficulty proceeds according to the type of activity; for instance, in a transfer activity of sitting to standing with support, progression can be obtained by incrementally lowering the height of the bench.

\section{Implementation of HABIT-ILE}

HABIT-ILE will be delivered by physiotherapists and students in their last year of physiotherapy bachelor's degree, acting as interventionists/children playmates. Before the intervention period, they will attend a 1-day course administered by two certified HABIT-ILE experts about the principles of HABIT-ILE. Each child will work with one or two interventionists during the HABIT-ILE, which will be performed following a camp-model for groups of 8-12 children. The intervention lasts 2 weeks for a total of 50 hours, as follows: 5 hours a day with 2 hours of break, that is, 3 hours of a.m. therapy, 2 hours of nap-break and 2 hours of p.m. therapy for 10 consecutive week days. This dosage is justified by pilot study results for early HABIT-ILE showing excellent improvement in fine and gross motor outcomes in children aged 1-4 years with unilateral $\mathrm{CP}$, along with good tolerance and no adverse events. ${ }^{16}$

In the original HABIT-ILE, the intervention is delivered in the absence of the parents. However, in this new context, aiming to promote the continuation of the activities while at home, the presence of the parents/caregivers will be allowed, and they may be involved in the games, although without intervening as interventionists. A daily work sheet will be maintained to record activities provided to each child. Activities and work material will be socio-culturally adapted, mostly using handcrafted, locally produced items that can be easily obtained even in remote areas of the country. The intervention will be performed at the clinic, in a playroom rehabilitation setting.

\section{Conventional therapy}

The conventional therapy delivered to children with CP in Benin is mostly oriented to goals defined by therapists that target the child's impairments, such as spasticity, reduced range of motion and delayed gross motor milestones. It generally consists of neurodevelopmentalbased therapy, gross motor and manual exercises, stretching, passive mobilisation and the like. The intervention will be delivered by five experienced neuropediatric physiotherapists with practice experience ranging from 7 to 17 years, and with the assistance of students in their last year of a physiotherapy bachelor's degree. Each child will work with one or two interventionists on a daily basis. A daily work sheet will be used to record the care provided to each child, allowing a detailed description of the particulars of this intervention for each child. Conventional therapy will be applied at the same dosage as for the HABIT-ILE group, that is, 5 hours per day during 10 consecutive weekdays for a total of 50 hours of therapy. The camp will be held at a clinic setting.

\section{Data monitoring committee}

The supervisors of each intervention will participate of the data monitoring committee and will screen each day for any adverse events, whether minor (such as falling during the therapies, muscles soreness) or major (such as moderate-to-severe falls with bruising or fractures) during therapies. However, no adverse events (neither minor nor major) related to HABIT-ILE therapy has been reported so far. ${ }^{14-16}$ Moreover, the conventional therapy is inherently unlikely to occasion any adverse events. 


\section{Fidelity}

Intervention fidelity for the HABIT-ILE therapy will be assured through ongoing coaching and supervision by the certified experts in HABIT-ILE along with its developer (YB) during the whole duration of the study. Moreover, physiotherapists involved in the conventional therapy will be kept unaware of the HABIT-ILE content during the implementation of the study, which shall occur at different sites. Therefore, children in each group will receive the intervention as planned, without interference from the other group.

\section{Outcome measurement}

Children's assessments include classification systems for descriptive purposes, and measurement tools to quantify changes after the interventions. Children in both groups will be evaluated within 1 week before the intervention, the week immediately after its conclusion and at 6-week follow-up. Assessments after the interventions will include only primary and secondary outcomes.

\section{Classification systems}

The Gross Motor Function Classification System (GMFCS) Expanded and Revised is a widely used and recommended tool to classify gross motor function with or without use of assistive devices in children with CP. The GMFCS instrument has established construct validity, and good reliability (test-retest and inter-raters) for children above 2 years old. ${ }^{28}$ The classification is based on a five-level ordinal scale from the least affected level I (walks without limitations) to the most affected level $\mathrm{V}$ (transported in a manual wheelchair). This study will include only children with levels III (W-sitting, use of hand-held mobility devices for short distance mobility) or IV (sitting with hand support; rolling for mobility) will be included.

The Manual Ability Classification System (MACS) for children over 4 years and the Mini-MACS for younger children classify how children self-initiate or need assistance when manipulating objects in daily life activities. ${ }^{28}{ }^{29}$ MACS is a well-known classification system of proven validity and reliability, also entailing a five-level ordinal scale ranging from level I (handles objects easily and successfully) to level V (cannot handle objects and has severely limited ability to perform even simple actions). This study will include children with MACS level I to III.

The Communication Function Classification System (CFCS) classifies children's communication performance, with respect to expression and reception with familiar and unfamiliar persons, irrespective of the method of communication (speech, gestures, behaviours, eye gaze, facial expressions or augmentative and alternative communication)..$^{30}$ Like the above-mentioned classifications, the CFCS is a valid and reliable metric (test-retest and inter-raters), ranked on an ordinal scale extending from level I (effective sender and receiver with unfamiliar and familiar partners) to level $\mathrm{V}$ (seldom effective sender and receiver, even with familiar partners) ${ }^{28} 30$

\section{Primary outcomes}

The Gross Motor Function Measure 66 items (GMFM-66) is a unidimensional and interval-scaling version of the previous GMFM-88 following the Rasch model used to measure changes in gross motor function in children with CP aged from 5 months to 16 years. ${ }^{31}$ The items are grouped in five subdomains: (i) lying and rolling, (ii) sitting, (iii) crawling and kneeling, (iv) standing and (v) walking, running and jumping. GMFM-66 has been proved valid, reliable and responsive to temporal changes in motor performance. ${ }^{31} 32$ The assessment is videorecorded and blind-scored on a four-level scale ranging from ' 0 ' (does not initiate) to ' 3 ' (completes). Raw GMFM-66 scores will be converted into linear interval scores with reference percentiles using proprietary software Gross Motor Ability Estimator.

The Both Hands Assessment (BoHA) measures the collaborative use of both hands in bimanual play by children with bilateral CP of MACS level I to III, aged from 18 months to 12 years. It is a Rasch-calibrated instrument consisting of 16 hierarchical items (11 unimanual items and 5 bimanual items). The BoHA is scored on a four-level scale ranging from ' 4 ' (effective) down to ' 1 ' (does not do). The total linear score depends on the (a)symmetric use of both hands. Its internal validity and discriminative validity with MACS have been shown, along with the Rasch calibration process. ${ }^{33}$ The assessment is video-recorded and scored by a blind certified rater.

\section{Secondary outcomes}

The Canadian Occupational Performance Measure (COPM) assesses both the performance and the satisfaction with the performance of functional goals rated on a 1-to-10 scale, with higher score reflecting better performance and satisfaction. Five goals are defined regarding daily life activities in self-care, productivity and leisure from among the expressed goals that the children aspire towards, but which currently present difficulties. ${ }^{34}$ The total scores for performance and the satisfaction are the mean scores for the five selected goals. The COPM is a valid, reliable and sensitive measure. ${ }^{34}$ Since this study will include younger children (from 24 to 59 months), goals will be set by parents/caregivers.

An adapted version of ACTIVLIM-CP questionnaire will measure each child's performance in daily life activities. The current ACTIVLIM-CP is a Rasch-built scale calibrated for children with CP in Europe, which is a reliable instrument that is sensitive to changes in global performance assessed through 43 daily activities involving upper and lower extremities. ${ }^{35}$ Parents of children score on a three-level scale (impossible, difficult, and easy) the difficulty their children experience in performing the listed activities.

The Perception-CP questionnaire, which aims to describe the perception of CP in parents/caregivers, includes items on knowledge of CP (causes, treatment approaches, developmental prognosis) and the impact of caregiving on social and family life. The content validity of the questionnaire 
was appraised by 10 physiotherapists who were instructed to evaluate the relevance and understanding of the items.

\section{Management of withdrawals}

Participants are to be informed during the recruitment process of their right to withdraw at any time without prejudice. Based on past experience, was accommodated an expected drop-out rate in estimating the sample size. Moreover, statistical analysis will be performed on an intention-to-treat basis to account for data lost due to withdrawal.

\section{Statistical analysis}

Data analysis will be performed with the intent-to-treat approach at a follow-up session. Baseline between-group comparisons will be tested with an independent t-test. Afterwards, a 2 (groups) $\times 3$ (testing sessions) factors repeated analysis of variance with repetition of the testing sessions will evaluate changes within and between the two groups at the conclusion of the interventions and at 6 weeks' follow-up. Outcome data at each session will be reported with means and $95 \%$ CIs. In the event that parametric analyses should be inappropriate, we shall use the non-parametric Mann-Whitney U test for betweengroup comparison and the Kruskal-Wallis test by ranks for within-group comparisons. Effect sizes will be computed for the magnitude of the efficacy of the interventions.

\section{ETHICS AND DISSEMINATION}

This study has been approved by the ethics committee of the rehabilitation department of the National Teaching Hospital Hubert Koutoukou Maga of Cotonou, Benin (approval decision: No01-2019/MS/CNHU-HKM/ CEI/CUMPR). All participants' parents/caregivers will provide their written informed consent. Data will be managed with confidentiality. Findings from this study will be disseminated through peer-reviewed scientific journals and at key national and international scientific events. The study is registered in the Pan African Clinical Trial Registry prior to children's recruitment, date of approval: 05 November 2019.

\section{Current status of the protocol}

This study is currently at the stage of interventions delivery with post-intervention assessments.

\section{Public/patient involvement statement}

Neither patients nor the general public were involved in the design, or conduct, or reporting, or dissemination plans of the study.

\section{DISCUSSION}

$\mathrm{CP}$ is more prevalent in low and middle-income countries such as Benin than in high-income countries; clinicallybased studies highlight the higher proportion of children in low and middle-income countries presenting a moderate-to-severe bilateral form of the disability. ${ }^{5-7}$
Nonetheless, access to rehabilitation care in sub-Saharan West Africa is limited by several factors, such as the sparsity of existing rehabilitation services, their concentration in urban settings, general lack of knowledge of caregivers about $\mathrm{CP}$ and issues related to stigmatisation and household finances. ${ }^{21}{ }^{23}$ Moreover, such rehabilitation care that is available in Africa remains based to a remarkable extent on non-evidence-based approaches, that is, passive hands-on treatments such as stretching, joint mobilisation and traditional neurodevelopmental therapies. ${ }^{24}{ }^{36}$ Given this prevailing context, the present study will investigate the efficacy of an intensive, 2 weeks rehabilitation intervention, HABIT-ILE, relative to that of a conventional intervention. Based on the scientific literature indicating a broader possibility for neuroplastic changes in younger children, we aimed this project at children with CP under the age of 5 years. ${ }^{3738}$ Confirmation of our several hypotheses should have a considerable impact on the quality of rehabilitation care for children with CP provided in Benin and other low and middle-income countries. We anticipate a shift towards goals-directed and child-active interventions, aiming to bring lasting benefits for children's autonomy, while having a positive impact on the perception of parents/caregivers towards the burden of CP. Conspicuous improvements after the HABIT-ILE intervention should encourage caregivers to continue the activities learnt at home.

\section{Author affiliations}

${ }^{1}$ Institute of Neuroscience, Université catholique de Louvain, Bruxelles, Belgium

${ }^{2}$ School of Physiotherapy, Université d'Abomey-Calavi, Faculté des Sciences de la

Santé, Cotonou, Benin

${ }^{3}$ Service de Kinésithérapie et d'Appareillage Orthopédique, Centre Hospitalier Universitaire Départemental de Borgou/Alibori, Parakou, Benin

${ }^{4}$ Centre Medico-social Sainte Elisabeth de la Trinité, Calavi, Benin

${ }^{5}$ Clinique universitaire de médecine physique et réadaptation, Centre National Hospitalier Universitaire Hubert Koutoukou Maga de Cotonou, Cotonou, Benin

${ }^{6}$ Physical and Occupational Therapy, Haute Ecole Louvain en Hainaut - Montigniessur-Sambre, Montignies-sur-Sambre, Belgium

${ }^{7}$ Département Ergothérapie, HE Vinci, Brussels, Belgium

Correction notice This article has been corrected since it first published. Affiliation for 'Julie Paradis' has been updated.

Acknowledgements Authors will be grateful to all participants to the study (therapists, caregivers and children). We are thankful to the physiotherapists Damienne Houekpetodji, Prunelle Togni and Roland Galokpo who will help in the delivery of interventions. We thank the reviewers for the constructive comments that improve the quality of our paper.

Contributors ESS, TGK and YB, contributed to the study design; ESS, SSA, LA, $\mathrm{SD}, \mathrm{HD}, \mathrm{DE}-\mathrm{K}, \mathrm{RA}, \mathrm{GS}$ and $\mathrm{YB}$ will be involved in the implementation of therapies; ESS will recruit the participants and perform the assessments; DE-K, RA, GS and JP will score the assessment videos; ESS and YB will contribute to data analysis. All authors have contributed to the drafting of this manuscript and have proved the final version of it.

Funding This work was supported by the Université Catholique de Louvain, Belgium, grant number (ADRI/CD/CAI2017-53).

\section{Competing interests None declared.}

Patient and public involvement Patients and/or the public were not involved in the design, or conduct, or reporting, or dissemination plans of this research.

Patient consent for publication Not applicable.

Provenance and peer review Not commissioned; externally peer reviewed. 
Open access This is an open access article distributed in accordance with the Creative Commons Attribution Non Commercial (CC BY-NC 4.0) license, which permits others to distribute, remix, adapt, build upon this work non-commercially, and license their derivative works on different terms, provided the original work is properly cited, appropriate credit is given, any changes made indicated, and the use is non-commercial. See: http://creativecommons.org/licenses/by-nc/4.0/.

\section{ORCID iDs}

Emmanuel Segnon Sogbossi http://orcid.org/0000-0003-4996-6909

Rodrigo Araneda http://orcid.org/0000-0002-0890-5504

\section{REFERENCES}

1 Rosenbaum P, Paneth N, Leviton A, et al. A report: the definition and classification of cerebral palsy April 2006. Dev Med Child Neurol Suppl 2007;109:8-14.

2 Arnould C, Bleyenheuft Y, Thonnard J-L. Hand functioning in children with cerebral palsy. Front Neurol 2014;5:48.

3 Graham HK, Rosenbaum P, Paneth N, et al. Cerebral palsy. Nat Rev Dis Primers 2016;2:15082.

4 Kakooza-Mwesige A, Andrews C, Peterson S, et al. Prevalence of cerebral palsy in Uganda: a population-based study. Lancet Glob Health 2017;5:e1275-82.

5 Sogbossi ES, Houekpetodji D, Kpadonou TG, et al. A cross-sectional study of the clinical profile of children with cerebral palsy in Benin, a West African low-income country. J Child Neurol 2019;34:842-50.

6 Bearden DR, Monokwane B, Khurana E, et al. Pediatric cerebral palsy in botswana: etiology, outcomes, and comorbidities. Pediatr Neurol 2016;59:23-9.

7 Ogoke CC, lloeje SO. Severity of motor dysfunction in children with cerebral palsy seen in Enugu, Nigeria. Pan Afr Med J 2017;27:154.

8 Novak I, Morgan C, Fahey M, et al. State of the evidence traffic lights 2019: systematic review of interventions for preventing and treating children with cerebral palsy. Curr Neurol Neurosci Rep 2020;20:3.

9 Bleyenheuft Y, Gordon AM. Hand-arm bimanual intensive therapy including lower extremities (HABIT-ILE) for children with cerebral palsy. Phys Occup Ther Pediatr 2014;34:390-403.

10 Muratori LM, Lamberg EM, Quinn L, et al. Applying principles of motor learning and control to upper extremity rehabilitation. $J$ Hand Ther 2013;26:94-103

11 Friel KM, Kuo H-C, Fuller J, et al. Skilled bimanual training drives motor cortex plasticity in children with unilateral cerebral palsy. Neurorehabil Neural Repair 2016;30:834-44.

12 Bleyenheuft Y, Dricot L, Ebner-Karestinos D, et al. Motor skill training may restore impaired corticospinal tract fibers in children with cerebral palsy. Neurorehabil Neural Repair 2020;34:533-46.

13 Sakzewski L, Ziviani J, Boyd RN. Efficacy of upper limb therapies for unilateral cerebral palsy: a meta-analysis. Pediatrics 2014;133:e175-204.

14 Bleyenheuft Y, Ebner-Karestinos D, Surana B, et al. Intensive upperand lower-extremity training for children with bilateral cerebral palsy: a quasi-randomized trial. Dev Med Child Neurol 2017;59:625-33.

15 Bleyenheuft Y, Arnould C, Brandao MB, et al. Hand and arm bimanual intensive therapy including lower extremity (HABIT-ILE) in children with unilateral spastic cerebral palsy: a randomized trial. Neurorehabil Neural Repair 2015;29:645-57.

16 Araneda R, Klöcker A, Ebner-Karestinos D, et al. Feasibility and effectiveness of HABIT-ILE in children aged 1 to 4 years with cerebral palsy: a pilot study. Ann Phys Rehabil Med 2021;64:101381.

17 Plasschaert VFP, Vriezekolk JE, Aarts PBM, et al. Interventions to improve upper limb function for children with bilateral cerebral palsy: a systematic review. Dev Med Child Neurol 2019;61:899-907.

18 Sakzewski L, Bleyenheuft Y, Boyd RN, et al. Protocol for a multisite randomised trial of hand-arm bimanual intensive training including lower extremity training for children with bilateral cerebral palsy: HABIT-ILE Australia. BMJ Open 2019;9:e032194.
19 Araneda R, Sizonenko SV, Newman CJ, et al. Protocol of changes induced by early hand-arm bimanual intensive therapy including lower extremities (e-HABIT-ILE) in pre-school children with bilateral cerebral palsy: a multisite randomized controlled trial. BMC Neurol 2020;20:243

20 Sakzewski L, Reedman S, McLeod K, et al. Preschool HABIT-ILE: study protocol for a randomised controlled trial to determine efficacy of intensive rehabilitation compared with usual care to improve motor skills of children, aged 2-5 years, with bilateral cerebral palsy. BMJ Open 2021;11:e041542.

21 Andrews C, Kakooza-Mwesige A, Almeida R, et al. Impairments, functional limitations, and access to services and education for children with cerebral palsy in Uganda: a population-based study. Dev Med Child Neurol 2020;62:454-62.

22 Sogbossi ES, Houekpetodji D, Kpadonou TG, et al. Mothers' perception of cerebral palsy in a low-income country of West Africa: a cross-sectional study. Disabil Rehabil 2021:1-8.

23 Paget A, Mallewa M, Chinguo D, et al. 'It means you are grounded' - caregivers' perspectives on the rehabilitation of children with neurodisability in Malawi. Disabil Rehabil 2016;38:223-34.

24 Okenwa W, Edeh A. A review of clinical presentation and physiotherapy management of cerebral palsy patients in Esut teaching hospital, Enugu, Nigeria. Afr Health Sci 2019;19:3085-90.

25 Zuurmond M, O'Banion D, Gladstone M, et al. Evaluating the impact of a community-based parent training programme for children with cerebral palsy in Ghana. PLoS One 2018;13:e0202096.

26 Donald KA, Samia P, Kakooza-Mwesige A, et al. Pediatric cerebral palsy in Africa: a systematic review. Semin Pediatr Neurol 2014;21:30-5.

27 Abdel Malek S, Rosenbaum P, Gorter JW. Perspectives on cerebral palsy in Africa: exploring the literature through the lens of the International classification of functioning, disability and health. Child Care Health Dev 2020;46:175-86.

28 Palisano RJ, Avery L, Gorter JW, et al. Stability of the gross motor function classification system, manual ability classification system, and communication function classification system. Dev Med Child Neurol 2018;60:1026-32.

29 Eliasson A-C, Ullenhag A, Wahlström U, et al. Mini-MACS: development of the manual ability classification system for children younger than 4 years of age with signs of cerebral palsy. Dev Med Child Neurol 2017;59:72-8.

30 Hidecker MJC, Paneth N, Rosenbaum PL, et al. Developing and validating the communication function classification system for individuals with cerebral palsy. Dev Med Child Neurol 2011;53:704-10.

31 Avery LM, Russell DJ, Raina PS, et al. Rasch analysis of the gross motor function measure: validating the assumptions of the Rasch model to create an interval-level measure. Arch Phys Med Rehabil 2003;84:697-705.

32 Wang $\mathrm{H}-\mathrm{Y}$, Yang $\mathrm{YH}$. Evaluating the responsiveness of 2 versions of the gross motor function measure for children with cerebral palsy. Arch Phys Med Rehabil 2006;87:51-6.

33 Elvrum A-KG, Zethræus B-M, Vik T, et al. Development and validation of the both hands assessment for children with bilateral cerebral palsy. Phys Occup Ther Pediatr 2018;38:113-26.

34 Pollock N, Baptiste S, Law M, et al. Occupational performance measures: a review based on the guidelines for the client-centred practice of occupational therapy. Can J Occup Ther 1990;57:77-81.

35 Paradis J, Arnould C, Thonnard J-L, et al. Responsiveness of the ACTIVLIM-CP questionnaire: measuring global activity performance in children with cerebral palsy. Dev Med Child Neurol 2018;60:1178-85.

36 Maharaj SS, White T-L, Kaka B. How are children with cerebral palsy managed in public hospitals of KwaZulu-Natal, South Africa? Physiother Theory Pract 2019:1-9.

37 Eyre JA. Corticospinal tract development and its plasticity after perinatal injury. Neurosci Biobehav Rev 2007;31:1136-49.

38 Martin JH, Friel KM, Salimi I, et al. Activity- and use-dependent plasticity of the developing corticospinal system. Neurosci Biobehav Rev 2007;31:1125-35. 